\title{
Prospective Observational Study of Factors Related to the Movement Subscale Score of the Japanese Orthopaedic Association Hip-Disease Evaluation Questionnaire Six Months After Total Hip Arthroplasty
}

Junya Sekita

Kitasato University

Naonobu Takahira ( $\nabla$ takahira@med.kitasato-u.ac.jp )

Kitasato University

Genki Iwamura

Zama General Hospital

Atsushi Kusaba

Zama General Hospital

Saiji Kondo

Zama General Hospital

Research Article

Keywords: Orthopaedic, Hip-Disease, JHEQ, THA

Posted Date: April 7th, 2021

DOI: https://doi.org/10.21203/rs.3.rs-377622/v1

License: (c) (i) This work is licensed under a Creative Commons Attribution 4.0 International License.

Read Full License 


\section{Abstract \\ Background}

The movement subscale score of the Japanese Orthopaedic Association Hip-Disease Evaluation Questionnaire (JHEQ) is reportedly lower than other subscale scores after total hip arthroplasty (THA). This study aimed to assess factors related to the movement subscale score of the JHEQ six months after THA.

\section{Methods}

Participants were 90 female patients with hip osteoarthritis who underwent THA. We examined clinical background factors and surgical information, and measured hip joint range of motion, lower extremity muscle strength, maximum walking speed, physical activity, Japanese Orthopaedic Association score, and JHEQ score. We also measured the number of steps and "fast walking time" as physical activity, and calculated the moderate intensity activity ratio. A stepwise logistic regression analysis was used to identify factors related to high and low movement subscale scores.

\section{Results}

Six months after surgery, the total score and all subscale scores of the JHEQ were significantly higher compared to preoperative scores $(p<0.01)$. The movement subscale score was lower compared to other subscale scores $(p<0.01)$. The stepwise multiple regression analysis revealed that preoperative movement subscale score (odds ratio: $1.26,95 \% \mathrm{Cl}: 1.08-1.47, p=0.003$ ), activities of daily living category of the Japanese Orthopaedic Association hip score (odds ratio: 1.66, 95\%Cl: 1.18-2.33, $\mathrm{p}=$ 0.004 ), and moderate intensity activity ratio (odds ratio: $1.05,95 \% \mathrm{Cl}: 1.00-1.11, \mathrm{p}=0.035$ ) are factors that determine high and low movement subscale scores.

\section{Conclusion}

The postoperative movement subscale score is related to not only the ability to conduct activities of daily living and preoperative movement subscale score, but also the moderate intensity activity ratio.

\section{Background}

Total hip arthroplasty (THA) is an effective treatment for reducing hip pain and restoring hip joint mobility [1]. In recent years, patient-based health-related quality of life (HRQOL) scales, in particular, the SF-36 and the Western Ontario and McMaster Universities Osteoarthritis Index (WOMAC), have been used to assess patient satisfaction after surgery [2, 3]. A meta-analysis assessing HRQOL scales reported improvements in postoperative scores compared to preoperative scores, reaching the same levels as those of control 
subjects within one to three years following THA [3]. Thus, THA can achieve good recovery results in terms of patient HRQOL.

While the aforementioned scales were generally developed according to the Western lifestyle [4], the Japanese Orthopaedic Association Hip-Disease Evaluation Questionnaire (JHEQ), developed by Matsumoto et al. [5] and contains questions regarding movements that require deep hip flexion, is designed to evaluate HRQOL in patients with hip osteoarthritis who have the Asian lifestyle. Improving JHEQ score is thus an important goal of postoperative rehabilitation in this patient population. A prospective study reported that the movement subscale score of the JHEQ remains lower than other subscale scores up to six months following THA, recovering only to about $50 \%$ of the maximum value [6]. Therefore, it is important to clarify factors related to the movement subscale score to examine appropriate rehabilitation methods.

Recent studies have reported that the postoperative movement subscale score was related to the Japanese Orthopaedic Association (JOA) hip score, as well as the preoperative movement subscale score $[6,7]$. In addition, since the movement subscale encompasses many movements requiring deep hip flexion, lower limb functions such as hip joint range of motion (ROM) and lower extremity muscle strength are also expected to be related. On the other hand, walking ability and physical activity (PA) are reportedly related to the physical function (PF) scale of the SF-36 and SF-8 PF in healthy subjects as well as THA patients [8-11]. Based on these reports, we hypothesized that not only the preoperative movement subscale score and JOA score, but also lower limb functions, walking ability, and PA are related to the JHEQ movement subscale score six months after surgery. Yet, no report to date has measured associations of these factors with the postoperative JHEQ movement subscale score.

The present study aimed to examine factors related to the movement subscale score of the JHEQ six months after THA.

\section{Participants And Methods Participants}

Participants were 90 female patients who were selected from among 527 patients with hip osteoarthritis who underwent THA at two hospitals in Japan between December 2015 and September 2017 (Fig. 1). Inclusion criteria were patients who (1) had primary unilateral cementless THA via an anterolateral minimally invasive approach; (2) were female; and (3) agreed with the purpose of this study. Since about $90 \%$ of our patients are female, we limited the subjects to females in order to minimize effects of gender differences in lifestyle. Exclusion criteria were (1) a history of lower limb or back surgery; (2) previously diagnosed painful orthopedic disease other than hip joint disease; (3) previously diagnosed hip osteoarthritis of the non-surgical side; (4) previously diagnosed mental disease or neuromuscular disease; and (5) postoperative complications such as fracture, dislocation, infection, or nerve paralysis. Four experienced physicians performed all surgeries. Surgery was performed with patients in the side 
lying position at a $60^{\circ}$ angle to the floor. The incision site was limited to between the femoris fascia lamina muscle and anterior fiber of the gluteus medius muscle. No navigation system was used during surgery. For cementless stems, the SL-Plus stem (Smith \& Nephew) was used in $74.4 \%$ of cases, Short Modular Femoral Hip System (Smith \& Nephew) in 12.2\%, and Global Tissue Sparing stem (Zimmer Biomet) in 7.8\%. For acetabular components, the R3 Acetabular System (Smith \& Nephew) was used in $57.8 \%$ of cases, Continuum Acetabular System (Zimmer Biomet) in 30.0\%, and G7 Acetabular System (Zimmer Biomet) in $7.8 \%$. The liner material was ceramic in $90.0 \%$ of cases, and cross-linked polyethylene in $10.0 \%$. The head material was ceramic in $93.3 \%$ of cases, and Oxinium (Smith \& Nephew) in $6.7 \%$. These components were determined by the attending physician based on the age of the patient and X-ray picture of the hip joint.

\section{Procedures}

This study was a six-month long prospective observational study. The study period was set at six months given the need to achieve sufficient recovery within the standard physical therapy period (i.e., 150 days after surgery), as defined in Japan. The JHEQ and JOA were measured before and six months after surgery. Physical functions were measured six months after surgery, and PA was measured five to six months after surgery. Postoperative rehabilitation was performed according to the clinical pathway at our institution. On the day after surgery, all patients were allowed to bear full weight and underwent inpatient rehabilitation. Rehabilitation consisted of gait exercises, passive ROM exercises, and muscle strengthening exercises. For gait exercises, patients used parallel bars in the beginning, and crutches or a walker from one week after surgery. By the time of discharge, all patients used a T cane during walking exercises. The length of hospital stay (LOS) according to the clinical pathway was three weeks. Most patients underwent outpatient rehabilitation roughly once a week after discharge, and performed activities such as getting up from the floor or ascending and descending stairs according to ability, in addition to inpatient rehabilitation.

\section{Measurements}

We collected participant background information and surgical information, including age, height, body weight, body mass index (BMI), duration of disease (years), LOS (days), intraoperative blood loss (ml), operation time (minutes), and the size of the head from medical charts. The size of the head was included due to a report suggesting that it affects range of motion and ADL [12]. Duration of disease was defined as the period from pain onset to surgery. We measured hip joint ROM (flexion, extension, abduction, abduction, external rotation, and internal rotation), lower extremity muscle strength on both sides (hip flexor, extensor, and abductor, and knee extensors and flexors), and maximum walking speed for physical functions, and PA, JOA score, and JHEQ score. All measurements were conducted by two physical therapists with a full understanding of measurement methods and practical experience. 
Lower extremity maximal isometric strength was measured using a hand-held dynamometer ( $\mu$ Tas F-1; Anima Corp., Tokyo, Japan). We referred to the measuring method proposed by Fukumoto et al [13]. For the assessment of hip flexors, extensors, and abductors, patients were positioned on a platform in a supine position. For the assessment of knee extensors and flexors, patients were positioned on a platform in a sitting position at a $90^{\circ}$ angle. After practice, muscle strength during 5 seconds of isometric contraction was measured twice, and the higher value shown on the hand-held dynamometer was used for analysis. In order to confirm the reliability of these measurement methods, we performed preliminary measurements and calculated the intra-class correlation coefficient (ICC) to assess intra-rater reliability. In all methods, ICC $(1,2)$ was over 0.95 . The length of lever arm $(m)$ was measured from the hip joint to the center of the sensor pad. Then, the torque to body weight ratio $(\mathrm{Nm} / \mathrm{kg})$ was calculated. ROM and lower extremity muscle strength were measured on both sides (surgical and non-surgical sides). JOA hip scores were measured according to the method prescribed by the JOA [14]. The ADL category of the JOA hip score was adopted as an indicator of ADL ability. The maximum 10-m walking speed was measured as an indicator of walking ability. If patients requested the use a cane, we permitted cane use. The number of measurements was set to two, and the maximum value $(\mathrm{m} / \mathrm{s})$ of the two measurements was used for the analysis of walking speed. For the measurement of PA, we used a digital pedometer with 3-axis acceleration sensors (TH-400; YAMASA, Tokyo, Japan) to measure the number of steps and "fast walking time." The validity and reliability of another device by the same manufacturer (EX-510; YAMAX, Tokyo, Japan), which uses the same algorithm, has been verified previously [15]. Fast walking time was measured at a cadence of $\geq 120$ (steps/minute) with this device. There is a strong correlation between walking cadence and exercise intensity [16], and faster walking time on this device indicates increased activity intensity. A cadence of $\geq 120$ (steps/min) corresponds to moderate or higher activity intensity ( $\geq$ 3-4 METs) [17]. Patients were instructed to wear the device on their body for 24 hours a day except when bathing or sleeping, for five to six months after surgery. After the measurement, we calculated the average number of steps (steps/day) and fast walking time (minutes/day) for the five-month period, excluding days when the device was not worn. Furthermore, the activity time per day was calculated from the number of steps (activity time per 1300 steps $=15$ minutes for elderly people [18]), and the moderate intensity activity ratio per day (\%) was calculated as the ratio of fast walking time to activity time per day.

JHEQ scores were determined according to the method described in previous studies [5-7]. The JHEQ consists of pain (28 points), movement (28 points), and mental (28 points) subscales, with a higher score indicating a better outcome. Patient dissatisfaction with their current condition and hip joint pain on each side were measured on a visual analog scale (VAS). The VAS for hip joint pain and questions were used to calculate subscale scores. The maximum total score for all subscales combined is 84 points.

\section{Statistical Analysis}

The Mann-Whitney $U$ test or $t$ test was used to assess changes in total and subscale scores of the JHEQ from before to six months after surgery. In addition, one-way analysis of variance and a posteriori Bonferroni test were used to assess differences in each subscale score six months after surgery. For analyses, patients were divided into two subgroups (high-score and low-score groups) based on the 
median movement subscale score. Next, the chi-square test, t-test, or the Mann-Whitney U test was used to compare clinical background factors, surgical information, physical functions, and PA between the two groups. Finally, a stepwise logistic regression analysis was performed to identify factors that determine high and low movement subscale scores, with high and low movement subscale scores as the objective variable, and items with a significant between-group difference as explanatory variables. We used the Hosmer and Lemeshow test as an index of goodness-of-fit. $P<0.05$ was considered statistically significant. R (ver. 3.4.1) was used for all analyses [19].

\section{Results}

Table 1 shows JHEQ scores and JOA hip scores for all patients before and six months after surgery. The total JHEQ score improved significantly from 23.2 points (27.6\%) before surgery to 65.7 points $(78.3 \%)$ after surgery $(p<0.01)$. Moreover, VAS for dissatisfaction and hip joint pain significantly decreased from before to after surgery $(p<0.01)$. All JHEQ subscale scores significantly improved from before to after surgery $(p<0.01)$. Significant differences were observed among the three subscale scores after surgery, with the movement subscale score being the lowest $(p<0.01)$. 
Table 1

JHEQ scores of all patients before and six months after surgery

Before surgery

Six months after surgery

p

VAS (surgical)

Dissatisfaction (mm)

94.0

$(78.0-98.0)$

5.5

$(2.0-12.0)$

$<0.001$

Pain (mm)

81.0

$(72.0-91.3)$

3.0

$(1.0-7.0)$

$<0.001$

JHEQ

Pain (surgical) (points)

$7.0 \quad(4.0-11.0)$

27.0

(24.0-28.0)

$<0.001$

Movement (surgical) (points)

5.0

$(1.8-7.3)$

18.0

$(14.0-22.0))^{\star \star} \ddagger$

$<0.001$

Mental (points)

$10.0 \quad(6.0-14.0)$

23.0

(20.0-28.0) $\ddagger$

$<0.001$

Total (points)

$23.0 \quad(14.0-30.5)$

68.0

$(58.8-74.0)$

$<0.001$

JOA hip score

Pain (surgical) (points)

$20.0 \quad(10.0-20.0)$

40.0

$(35.0-40.0)$

$<0.001$

ROM (surgical) (points)

$13.0 \quad(10.0-14.0)$

15.0

(14.0-16.0)

$<0.001$

Walk (points)

$10.0 \quad(10.0-15.0)$

20.0

(18.0-20.0)

$<0.001$

ADL (points)

14.0

(12.0-16.0)

18.0

$(16.0-20.0)$

$<0.001$

Total (points)

$54.0 \quad(45.0-62.3) \quad 90.0$

(87.0-94.0)

$<0.001$

Values are expressed as mean \pm SD or median (lower quartile - upper quartile)

VAS: visual analog scale, JHEQ: Japanese Orthopaedic Association Hip-Disease Evaluation Questionnaire

$\star *: p<0.01$, Significant difference compared to mental subscale score

‡: $p<0.01$, Significant difference compared to pain subscale score

The median movement subscale score was 18 points, and the mean movement subscale score was 17.2 points. Patients who scored $>18$ points were classified into the high-score group (42 patients), and the remaining patients into the low-score group (48 patients). The total JHEQ score was significantly higher in the high-score group compared to the low-score group $(p<0.01)$. Moreover, subscale scores were all significantly higher in the high-score group compared to the low-score group $(p<0.01)$.

Table 2 shows patient clinical background factors and surgical information by group. No significant differences were observed between the two groups. 
Table 2

Clinical background factors and surgical information

\begin{tabular}{|c|c|c|c|c|c|c|c|}
\hline \multirow[b]{2}{*}{ Age (years) } & \multicolumn{3}{|c|}{$\begin{array}{l}\text { Low score group } \\
(n=48)\end{array}$} & \multicolumn{3}{|c|}{$\begin{array}{l}\text { High score group } \\
(n=42)\end{array}$} & \multirow{2}{*}{$\begin{array}{l}\mathbf{p} \\
0.306\end{array}$} \\
\hline & 64.3 & \pm & 8.2 & 64.3 & \pm & 7.9 & \\
\hline Height (cm) & 154.4 & \pm & 5.1 & 154.4 & \pm & 4.9 & 0.206 \\
\hline Body weight (kg) & 55.6 & \multicolumn{2}{|c|}{$(49.8-59.2)$} & 51.6 & \multicolumn{2}{|c|}{$(49.4-57.3)$} & 0.158 \\
\hline BMI (kg/m2) & 23.0 & \multicolumn{2}{|c|}{$(21.6-24.7)$} & 23.4 & \multicolumn{2}{|c|}{$(20.8-24.0)$} & 0.278 \\
\hline LOS (day) & 20.7 & \pm & 6.9 & 20.7 & \pm & 5.5 & 0.446 \\
\hline $\begin{array}{l}\text { Duration of disease } \\
\text { (years) }\end{array}$ & 5.0 & \multicolumn{2}{|c|}{$(3.0-12.3)$} & 5.0 & \multicolumn{2}{|c|}{$(2.0-10.0)$} & 0.456 \\
\hline $\begin{array}{l}\text { Operation time } \\
\text { (minutes) }\end{array}$ & 71.5 & \multicolumn{2}{|c|}{$(61.5-81.3)$} & 68.0 & \multicolumn{2}{|c|}{$(60.0-80.5)$} & 0.512 \\
\hline Intraoperative blood loss (ml) & 205.0 & \multicolumn{2}{|c|}{$(177.5-300.0)$} & 200.0 & \multicolumn{2}{|c|}{$(113.8-300.0)$} & 0.257 \\
\hline $\begin{array}{l}\text { Size of the head } \\
(32 \mathrm{~mm} / 36 \mathrm{~mm})\end{array}$ & \multicolumn{3}{|l|}{$22 / 26$} & \multicolumn{3}{|l|}{$25 / 17$} & 0.195 \\
\hline \multicolumn{8}{|c|}{ Values are expressed as mean \pm SD or median (lower quartile - upper quartile) } \\
\hline \multicolumn{8}{|c|}{ BMI: Body mass index, LOS: Length of stay } \\
\hline No significant difference was & ve & & 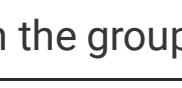 & & & & \\
\hline
\end{tabular}

Table 3 shows JOA hip scores, hip joint ROM, physical functions, and PA of patients in both groups six months after surgery. ADL and gait categories of the JOA hip score, hip extensor muscle strength on nonsurgical side, hip extension ROM on non-surgical side, hip abduction ROM on surgical side, maximum 10$\mathrm{m}$ walking speed, fast walking time, and moderate intensity activity ratio were significantly higher in the high-score group compared to the low-score group $(p<0.05)$. No significant differences were observed in other measurements. 
Table 3

JOA hip score, hip joint ROM, physical function, and PA in both groups

Low score group High score group

p

VAS (surgical)

Dissatisfaction (mm)

$\begin{array}{lllll}6.0 & (1.0-15.3) & 5.0 & (2.0-9.0) & 0.467\end{array}$

Pain (mm)

3.0

$(1.0-10.3)$

2.0

$(0.0-5.0)$

0.114

JHEQ

Pain (surgical) (points)

Before
surgery

6.0

$(3.8-10.3)$

7.5

(5.0-

11.0)

0.145

$\begin{array}{llllll}\begin{array}{l}\text { After } \\ \text { surgery }\end{array} & 26.5 & (22.0-28.0) & 28.0 & (25.3- & 0.027 \\ 28.0) & & & \end{array}$

Movement (surgical)

(points)

Before

surgery

$3.5 \quad(1.0-6.0)$

6.5

$(5.0-9.0)$

$<$

After

surgery

14.0

$(11.5-17.0) \quad 22.0$

(21.0-

23.8)

0.001

Mental (points)

Before

surgery

7.0

$(4.5-12.0)$

11.0

(8.0-

15.0)

$<$

After

surgery

22.0

$(18.8-24.3) \quad 26.0$

(22.3-

28.0)

Total (points)

\begin{tabular}{l}
$\begin{array}{l}\text { Before } \\
\text { surgery }\end{array}$ \\
\hline $\begin{array}{l}\text { After } \\
\text { surgery }\end{array}$
\end{tabular}

18.5

$(10.8-25.5)$

25.0

(19.0-

33.8)

$<$

61.0

$(54.0-68.0)$

74.5

$(71.0-$

0.001

JOA hip score

Pain (surgical side) (points)

40.0

$(35.0-40.0)$

40.0

(35.0-

40.0)

0.667

ROM (surgical side) (points)

15.5

(14.8-16.0)

15.0

(14.0-

16.0)

Walk (points)

18.0

(18.0-20.0)

20.0

(19.5-

20.0)

ADL (points)

16.0

$(16.0-20.0)$

20.0

(18.0-

20.0)

Total (points)

88.0

(83.5-93.3)

91.0

(89.3-
$94.0)$

0.317

0.001 
Hip joint ROM

Flexion $\left(^{\circ}\right)$

\begin{tabular}{lclccc} 
surgical & 100.0 & $\begin{array}{l}(90.0- \\
105.0)\end{array}$ & 100.0 & $\begin{array}{l}(91.3- \\
108.8)\end{array}$ & 0.608 \\
\hline non-surgical & 102.5 & $\begin{array}{l}(100.0- \\
110.0)\end{array}$ & 100.0 & $\begin{array}{l}(91.3- \\
110.0)\end{array}$ & 0.338 \\
& & & &
\end{tabular}

Extension $\left({ }^{\circ}\right)$

surgical

10.0

$(5.0-10.0)$

10.0

(5.0-

10.0)

0.166

non-surgical

10.0

$(5.0-10.0)$

10.0

(10.0-

10.0)

Abduction $\left({ }^{\circ}\right)$

surgical

20.0

$(20.0-30.0)$

25.0

(20.0-

30.0)

$\begin{array}{lllll}\text { non-surgical } & 25.0 \quad(20.0-30.0) & 25.0 & (20.0- \\ 30.0)\end{array}$

Adduction ( $\left.{ }^{\circ}\right)$

surgical

10.0

$(10.0-15.0)$

12.5

$(10.0-$
$15.0)$

0.014

non-surgical

10.0

$(10.0-15.0)$

15.0

(10.0-

0.077

External rotation $\left({ }^{\circ}\right)$

surgical
non-surgical

20.0

$(20.0-25.0)$

25.0

15.0)

surgical

25.0

$(20.0-30.0)$

22.5

$(20.0-$

0.080

Internal rotation $\left(^{\circ}\right)$

\begin{tabular}{lcccc} 
surgical & 27.5 & $(20.0-35.0)$ & 27.5 & $\begin{array}{l}(21.3- \\
35.0)\end{array}$ \\
\hline non-surgical & 25.0 & $(20.0-30.0)$ & 25.0 & $\begin{array}{l}(25.0- \\
30.0)\end{array}$
\end{tabular}

(20.0-

0.820 30.0)

0.631

Lower extremity muscle strength

Hip flexor

$(\mathrm{Nm} / \mathrm{kg})$

Hip extensor ( $\mathrm{Nm} / \mathrm{kg})$

Hip abductor $(\mathrm{Nm} / \mathrm{kg})$ surgical

0.82

$(0.69-1.04)$

0.85

(0.791.09)

$\begin{array}{llllllll}\text { non-surgical } & 1.06 & \pm & 0.27 & 1.13 & \pm & 0.25 & 0.199 \\ \text { surgical } & 1.09 & \pm & 0.31 & 1.18 & \pm & 0.39 & 0.237 \\ \text { non-surgical } & 1.15 & (0.86-1.36) & 1.27 & \begin{array}{l}(0.99- \\ 1.54)\end{array} & 0.048\end{array}$

surgical

$0.91 \quad \pm \quad 0.22$

$0.96 \pm 0.25$

0.293

Values are expressed as mean $\pm S D$ or median (lower quartile - upper quartile) JOA: Japanese Orthopaedic Association hip score, ROM: range of motion, PA: physical activity 


\begin{tabular}{|c|c|c|c|c|c|c|c|c|}
\hline & & \multicolumn{3}{|c|}{ Low score group } & \multicolumn{3}{|c|}{ High score group } & \multirow{2}{*}{$\begin{array}{l}\mathbf{p} \\
0.452\end{array}$} \\
\hline & non-surgical & 1.00 & & $3-1.10)$ & 1.01 & & & \\
\hline \multirow[t]{2}{*}{ Knee extensor (Nm/kg) } & surgical & 1.10 & \pm & 0.27 & 1.16 & \pm & 0.26 & 0.328 \\
\hline & non-surgical & 1.25 & \pm & 0.32 & 1.28 & \pm & 0.29 & 0.605 \\
\hline \multirow[t]{2}{*}{ Knee flexsor $(\mathrm{Nm} / \mathrm{kg})$} & surgical & 0.61 & \pm & 0.20 & 0.63 & \pm & 0.19 & 0.750 \\
\hline & non-surgical & 0.67 & & $4-0.80)$ & 0.68 & & & 0.357 \\
\hline \multicolumn{2}{|c|}{ Maximum walking speed (m/sec) } & 1.68 & & $5-1.82)$ & 1.77 & & & 0.006 \\
\hline \multicolumn{9}{|l|}{ PA } \\
\hline \multicolumn{2}{|l|}{ Number of steps (steps) } & 4241 & \multicolumn{2}{|c|}{$\begin{array}{l}(3332- \\
5707)\end{array}$} & 5094 & \multicolumn{2}{|c|}{$\begin{array}{l}(3620- \\
7315)\end{array}$} & 0.061 \\
\hline \multicolumn{2}{|l|}{ Activity time (min) } & 48.9 & \multicolumn{2}{|c|}{$(38.4-65.9)$} & 58.8 & \multicolumn{2}{|c|}{$\begin{array}{l}(41.8- \\
84.4)\end{array}$} & 0.061 \\
\hline \multicolumn{2}{|c|}{ Fast walking time(min/day) } & 9.5 & \multicolumn{2}{|c|}{$(4.8-14.2)$} & 16.0 & \multicolumn{2}{|c|}{$\begin{array}{l}(10.0- \\
26.8)\end{array}$} & $\begin{array}{l}< \\
0.001\end{array}$ \\
\hline \multicolumn{2}{|c|}{ Moderate intensity activity ratio (\%) } & 17.3 & \multicolumn{2}{|c|}{$(11.6-27.7)$} & 26.9 & \multicolumn{2}{|c|}{$\begin{array}{l}(22.4- \\
37.8)\end{array}$} & $\begin{array}{l}< \\
0.001\end{array}$ \\
\hline \multicolumn{9}{|c|}{ Values are expressed as mean \pm SD or median (lower quartile - upper quartile) } \\
\hline
\end{tabular}

Table 4 shows the results of stepwise logistic regression analysis with high and low postoperative movement subscale scores as the objective variable, and age and measured items with a significant between-group difference as explanatory variables. The preoperative movement subscale score (OR: 1.26, 95\%Cl: $1.08-1.47, p=0.003)$, ADL category of the JOA hip score (OR: $1.66,95 \% \mathrm{Cl}: 1.18-2.33, \mathrm{p}=0.004$ ), and moderate intensity activity ratio (OR: $1.05,95 \% \mathrm{Cl}: 1.00-1.11, \mathrm{p}=0.035)$ were extracted as factors that determine high and low movement subscale scores in the stepwise multiple regression analysis. Four explanatory variables were entered into the prediction model, yielding a minimum number of event generation samples necessary for logistic regression analysis of 40 people [20]. Since there were 42 people in the high-score group, the sample size was sufficient. Variance inflation factor was in the range of 1.08 to 1.36, and multicollinearity was not observed. Results of the Hosmer and Lemeshow test showed no issues with model adaptation $(p=0.232)$. 
Table 4

Results of stepwise logistic regression analysis

\begin{tabular}{|c|c|c|c|c|c|}
\hline \multirow[t]{2}{*}{ Explanatory variables } & \multirow[t]{2}{*}{ Standard error } & \multirow{2}{*}{$\begin{array}{l}\text { Adjusted } \\
\text { OR }\end{array}$} & \multicolumn{2}{|c|}{$95 \% \mathrm{Cl}$ of OR } & \multirow[t]{2}{*}{$\mathbf{p}$} \\
\hline & & & Lower & Upper & \\
\hline Age & 0.04 & 1.07 & 0.92 & 1.16 & 0.079 \\
\hline Preoperative movement subscale score & 0.08 & 1.26 & 1.08 & 1.47 & 0.002 \\
\hline ADL category of JOA hip score & 0.18 & 1.66 & 1.18 & 2.33 & 0.004 \\
\hline Moderate intensity activity ratio & 0.03 & 1.05 & 1.00 & 1.11 & 0.035 \\
\hline
\end{tabular}

\section{Discussion}

To the best of our knowledge, this is the first report to clarify factors related to the movement subscale score of the JHEQ six months after THA. The preoperative movement subscale score, ADL category of the JOA hip score, and moderate intensity activity ratio were extracted as independent factors determining high and low movement subscale scores. These findings suggest that the postoperative movement subscale score is related not only to ADL ability and preoperative movement subscale scores, but also the moderate intensity activity ratio.

Postoperative pain and mental subscale scores of the JHEQ recovered to $90.8 \%$ and $81.9 \%$ of the maximum score, respectively, whereas the postoperative movement subscale score only recovered to $62.3 \%$, which is significantly lower. A recent study that used the JHEQ to evaluate clinical outcomes of patients six months after THA reported that the postoperative movement subscale score was $52.9 \%$ of the maximum score, which was significantly lower than other subscale scores [6]. Our results are consistent with this report. The SF-36 and WOMAC PF scores reportedly recover to about $80 \%$ of the maximum value by six to eight months postoperatively [21]. Compared with these results, the recovery of the JHEQ movement subscale score is considerably lower. The movement subscale is composed of activities related to lifestyle that require deep flexion of the hip joint (e.g., using a squatting pan toilet, or getting up from the floor) [5-7]. Our results suggest that patients have difficulty with activities requiring deep flexion of the hip joint following THA, even after six months, and that an evaluation using the JHEQ could identify this problem.

Since the postoperative movement subscale score of the JHEQ did not have a normal distribution, logistic regression analysis was used after conversion to qualitative data. Useful clinical criteria were unclear, and thus we adopted median score as the criterion for grouping. In order to determine whether the median score used for grouping was valid, we compared our results with those of previous studies. For instance, Fukui et al. reported a mean score of 14.8 for the movement subscale score of the operative side at six months after THA [6]. Our corresponding score (median: 18.0, mean value: 17.7) was higher. One 
potential reason for this discrepancy is that the study by Fukui et al. adopted patients who underwent bilateral THA, whereas our subjects underwent unilateral THA. We also compared our results with those from a study by Fukumoto et al., which used a postoperative protocol and inclusion criteria more similar to ours [13]. They reported a mean score of 18.1 for the ADL category of the JOA hip score of the operative side six months after THA, which was consistent with our postoperative score. Subjects of the present study were considered to have comparable ADL ability compared to subjects in the study by Fukumoto et al. Since the ADL category of the JOA hip score and movement subscale score of the JHEQ are strongly correlated [7], our postoperative movement subscale score is unlikely to be biased relative to general subjects who undergo primary unilateral THA. Based on the above, we considered it valid to use median score as the criterion to identify factors that predict high and low movement subscale scores.

The stepwise logistic regression analysis with high and low postoperative movement subscale scores as the objective variable and items with a significant between-group difference as explanatory variables revealed that preoperative movement subscale score, ADL category of the JOA hip score, and moderate intensity activity ratio were factors that determine high and low movement subscale scores. These results are in line with a previous study reporting that the ADL ability and preoperative conditions are factors related to the movement subscale score. A study examining relationships between the JHEQ movement subscale score and other existing scales in patients with hip joint disease reported that the JHEQ movement subscale score is related to all subscale scores of the JOA, in particular, the score of the ADL category [7]. Although the JHEQ is used to assess patient-based QOL, the movement subscale score reflects the movement ability evaluated by a physician or physical therapist. In addition, according to previous studies, preoperative physical function is the best predictive index of postoperative physical function $[22,23]$, and the preoperative movement subscale score strongly affects scores obtained six months postoperatively [6].

We also had some new findings. Previous studies reported that moderate intensity activity time is related to HRQOL scales, especially the PF scale of the SF-36 and SF-8 [8-10]. Aoyagi et al. reported significant relationships between physical activity at intensity $>3$ METs per day (i.e., moderate-intensity activity) and the SF-36 PF in community-dwelling elderly people [8]. On the other hand, whether the moderate intensity activity ratio is related to the postoperative movement subscale score is unknown. Therefore, our finding that the postoperative movement subscale score is related to PA, especially the moderate intensity activity ratio, six months after surgery offers a new perspective. A previous study investigating the intensity of PA six months after surgery reported that light-intensity activity time reaches a level equivalent to that of healthy subjects, but moderate-intensity activity time is lower than that of healthy subjects [10]. In other words, the ability to perform moderate-intensity activities may not be fully recovered six months after surgery. In the present study, we found that the postoperative movement subscale score and the moderate intensity activity ratio are related. Moreover, the intensity of PA may be restricted, which could in turn suppress the improvement of the postoperative movement subscale score.

The JHEQ movement subscale is mainly composed of activities that require deep flexion of the hip joint, and the ROM of the hip joint is thought to strongly affect these activities [12]. However, the present study 
showed no significant difference between the two groups with respect to hip flexion ROM, and hip joint ROM was not extracted as a factor determining high and low movement subscale scores. Given that the average hip flexion ROM was about $95^{\circ}$ in our patients, flexion range might not be a major factor that limits activities that require deep flexion of the hip joint.

The preoperative movement subscale score, ADL category of the JOA hip score, and moderate intensity activity ratio were extracted as independent factors determining high and low movement subscale scores. In order to improve the movement subscale score after THA, training to acquire the ability to perform moderate-intensity activities will be needed, in addition to maintaining preoperative functions and incorporating various $A D L$ exercises into postoperative rehabilitation.

This study has several limitations. First, we investigated outcomes only after six months following THA; thus, the relevance of the present study is unclear with respect to outcomes after one year or longer. Second, the statistical methods which used median score as the criterion for grouping could have increased $\beta$ error [24]. Third, we used only the ADL category of the JOA hip score to assess ADL ability, and thus the ability of patients to remove socks or clip nails, for example, could not be analyzed. In the future, a long-term study will be necessary to assess all actions that make up the JHEQ.

\section{Conclusion}

We assessed factors related to the movement subscale score of the JHEQ six months after THA. The postoperative movement subscale score is related not only to ADL ability and preoperative movement subscale scores, but also the moderate intensity activity ratio.

\section{List Of Abbreviations}

THA

Total hip arthroplasty

HRQOL

health-related quality of life

JHEQ

Japanese Orthopaedic Association Hip-Disease Evaluation Questionnaire

JOA

Japanese Orthopaedic Association

ROM

range of motion

PA

physical activity

PF

physical function

BMI 
body mass index

LOS

length of hospital stay

ICC

intra-class correlation coefficient

OR

Odds ratio

$\mathrm{Cl}$

Confidence interval

\section{Declarations}

\section{Ethics approval and consent to participate}

This study complies with the ethical standards of the Declaration of Helsinki (1964) and its subsequent amendments and was approved by the Ethics Review Committees of Ebina General Hospital and Zama General Hospital (No. 207). Each patient provided written informed consent to participate in this study.

\section{Consent for publication}

Not applicable

\section{Availability of data and materials}

The datasets used and/or analyzed during the current study are available from the corresponding author on reasonable request.

\section{Competing interests}

The authors declare that they have no competing interests.

\section{Funding}

Research funds from our institution were used to support this study.

\section{Authors' contributions}

NT contributed to the study conception. JS and NT contributed to the study design. AK and SK performed total hip arthroplasty. SK provided a research environment. JS and GI contributed to the acquisition of data. Data analysis was mainly performed by JS, NT, and GI. JS, NT, and GI contributed to the interpretation of data and consideration of adequacy for data. JS and NT were major contributors in writing the manuscript and all authors contributed to drafting of the manuscript for important intellectual content. All authors read and approved the final version of the manuscript and have agreed to be personally accountable for their own contributions. 


\section{Acknowledgements}

We thank all individuals who participated in this study.

\section{References}

1. Siopack JS, Jergesen HE. Total hip arthroplasty. West J Med 1995; 162: 243-249.

2. Brokelman RB, van Loon CJ, Rijnberg WJ. Patient versus surgeon satisfaction after total hip arthroplasty. J Bone Joint Surg Br. 2003; 85: 495-498.

3. Shan L, Shan B, Graham D, Saxena A. Total hip replacement: a systematic review and meta-analysis on mid-term quality of life. Osteoarthritis Cartilage. 2014; 22: 389-406.

4. Beaton DE, Schemitsch E. Measures of health-related quality of life and physical function. Clin Orthop Relat Res. 2003; 413: 90-105.

5. Matsumoto T, Kaneuji A, Hiejima Y, Sugiyama H, Akiyama H, Atsumi T, Ishii M, Izumi K, Ichiseki T, Ito H, Okawa T, Ohzono K, Otsuka H, Kishida S, Kobayashi S, Sawaguchi T, Sugano N, Nakajima I, Nakamura S, Hasegawa Y, Fukuda K, Fujii G, Mawatari T, Mori S, Yasunaga Y, Yamaguchi M. Japanese Orthopaedic Association Hip Disease Evaluation Questionnaire (JHEQ): a patient-based evaluation tool for hip-joint disease. The Subcommittee on Hip Disease Evaluation of the Clinical Outcome Committee of the Japanese Orthopaedic Association. J Orthop Sci. 2012; 17: 25-38.

6. Fukui K, Kaneuji A, Sugimori T, Ichiseki T, Matsumoto T, Hiejima Y. Clinical assessment after total hip arthroplasty using the Japanese Orthopaedic Association Hip-Disease Evaluation Questionnaire J Orthop. 2015; 12: 31-36.

7. Seki T, Hasegawa Y, Ikeuchi K, Ishiguro N, Hiejima Y. Reliability and validity of the Japanese Orthopaedic Association hip disease evaluation questionnaire (JHEQ) for patients with hip disease. J Orthop Sci. 2013; 18: 782-787.

8. Aoyagi Y, Park H, Park S, Shephard RJ. Habitual physical activity and health-related quality of life in older adults: interactions between the amount and intensity of activity (the Nakanojo Study). Qual Life Res. 2010; 19: 333-338.

9. Anokye NK, Trueman P, Green C, Pavey TG, Taylor RS. Physical activity and health related quality of life. BMC Public Health. 2012; 12: 624.

10. Fujita K, Makimoto K, Tanaka R, Mawatari M, Hotokebuchi T. Prospective study of physical activity and quality of life in Japanese women undergoing total hip arthroplasty. J Orthop Sci 2013; 18: 4553.

11. Sliwinski M, Sisto S. Gait, quality of life, and their association following total hip arthroplasty. J Geriatr Phys Ther. 2006; 29: 10-17.

12. Matsushita I, Morita Y, Ito Y, Gejo R, Kimura T. Activities of daily living after total hip arthroplasty. Is a 32-mm femoral head superior to a 26-mm head for improving daily activities? Int Orthop. 2011; 35: 25-29. 
13. Fukumoto $\mathrm{Y}$, Ohata $\mathrm{K}$, Tsukagoshi R, Kawanabe K, Akiyama H, Mata T, Kimura M, Ichihashi N. Changes in hip and knee muscle strength in patients following total hip arthroplasty. J Jpn Phys Ther Assoc 2013; 16: 22-27.

14. Kuribayashi M, Takahashi KA, Fujioka M, Ueshima K, Inoue S, Kubo T. Reliability and validity of the Japanese Orthopaedic Association hip score. J Orthop Sci 2010; 15: 452-458.

15. Crouter SE, Schneider PL, Karabulut M, Bassett DR. Validity of 10 electronic pedometers for measuring steps, distance, and energy cost. Med Sci Sports Exerc. 2003; 35: 1455-1460.

16. Tudor-Locke C, Rowe DA. Using cadence to study free-living ambulatory behaviour. Sports Med. 2012; 42: 381-398.

17. Rowe DA, Welk GJ, Heil DP, Mahar MT, Kemble CD, Calabró MA, Camenisch K. Stride rate recommendations for moderate-intensity walking. Med Sci Sports Exerc. 2011; 43: 312-318.

18. Hearth Japan 21. [Internet]. Targeted value of Hearth Japan 21. http://www1.mhlw.go.jp/topics/kenko21_11/t2a.html (Accessed Apr. 4, 2016). (in Japanese).

19. Kanda Y. Investigation of the freely available easy-to-use software 'EZR' for medical statistics. Bone Marrow Transplant. 2013; 48: 452-458.

20. Peduzzi P, Concato J, Kemper E, Holford TR, Feinstein AR. A simulation study of the number of events per variable in logistic regression analysis. J Clin Epidemiol. 1996; 49: 1373-1379.

21. Vissers MM, Bussmann JB, Verhaar JA, Arends LR, Furlan AD, Reijman M. Recovery of physical functioning after total hip arthroplasty: systematic review and meta-analysis of the literature. Phys Ther. 2011; 91: 615-629.

22. Nankaku M, Tsuboyama T, Akiyama H Kakinoki R, Fujita Y, Nishimura J, Yoshioka Y, Kawai H, Matsuda S. Prediction of ambulation ability following total hip arthroplasty. J Orthop Sci $2011 ; 16$ : 359-363.

23. Judd DL, Dennis DA, Thomas AC, Wolfe P, Dayton MR, Stevens-Lapsley JE. Muscle strength and functional recovery during the first year after THA. Clin Orthop Relat Res 2014; 472: 654-664.

24. Derek D, Blakeley B, Preacher KJ A researcher's guide to regression, discretization, and median splits of continuous variables. Journal of Consumer Psychology 2015; 25: 666-678

\section{Figures}


527 patients with hip osteoarthritis underwent THA at two hospitals between December 2015 and September 2017

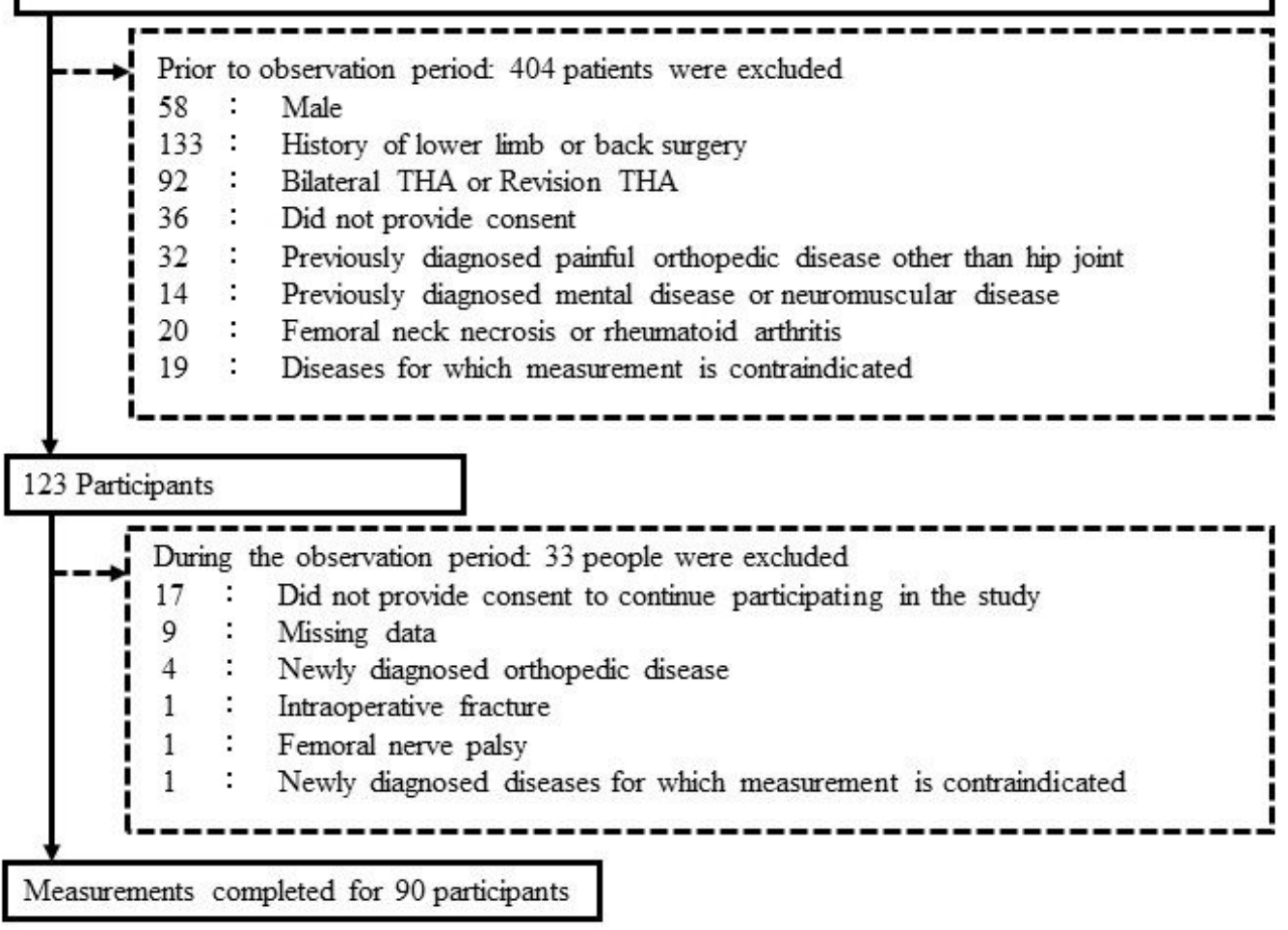

\section{Figure 1}

Flowchart for participant selection. 